\title{
Infrasonic fluidic oscillator for use in anti-terrorist warfare
}

\author{
V. Tesařr ${ }^{1}$ \& E. Smyk ${ }^{2}$ \\ ${ }^{1}$ Institute of Thermomechanics ASCR, Czech Republic \\ ${ }^{2}$ UTP University of Science and Technology, Poland
}

\begin{abstract}
The authors investigated a new approach to improving the effectiveness of detecting trace amounts of illegal substances, such as those on clothing of persons who have come into recent contact with explosives. An annular synthetic jet, generated by a simple and inexpensive non-moving-part fluidic oscillator operating at infrasonic frequencies protects the sample on its path - from the clothing surface to the inlet in an analyser - and prevents its dilution and escaping into the atmosphere.
\end{abstract}

Keywords: anti-terrorist warfare, synthetic jet, impinging jet, infrasonics.

\section{Introduction}

Terrorism is among the severest problems our civilisation is now facing. The success of terrorists is due to the economic factors favouring them [1]. It is cheaper by several orders of magnitude to launch a terrorist attack than to prevent it by deploying, maintaining, and manning large-scale protection systems and organisations. Prevention requires detection and early warning so effective that potential attackers are deterred. Among the detection and screening methods, the most expeditious is discovering on a person's clothing even the minutest traces of illegal substances - ranging from detecting concealed explosives to single out those persons who were merely present in a room where the illegal materials were handled. Standard detection uses trained dogs but this will not suffice in the near future. It is not fully reliable and training and keeping dogs by dedicated personnel at the vast numbers likely to be needed is economically untenable. Replacement by technological solution is necessary and of particular promise are ideas offered by fluidics - the technique of handling and controlling 
liquid and gas flows without the action of moving components. Fluidic solutions are inexpensive and in collaboration with microelectronics provide means shifting the current economic disbalance at least slightly away from terrorism.

\section{The task}

Substituting the dog-based detection activities by a technological solution needs solving several development steps. First, the detection has to be extremely sensitive. Dogs on average can detect explosives at concentrations in air as low as $1 \mathrm{ppb}$ (parts per billion) - depending on composition and vapour pressure of the particular explosive. Nevertheless, sufficiently sensitive multi-spectral sensors became already commercially available at a reasonable price $[2,3]-$ an achievement mainly thanks to microelectronics but in a non-negligible measure also to microfluidics [2].

Effort now focuses on efficiency of sample acquisition and delivery to the sensor. Progress in this direction can significantly relax the demands placed on the analyser sensitivity. Two areas are in need of improvement: (a) It is necessary to remove more effectively the detected substances from the interrogated surface. The limit is posed by the demand of the procedures being not overly unpleasant to public. (b) Also handling the samples must be more effective, in particular preventing their dilution. The main problem is anthropometric variance of screened persons. It results in a gap the sample has to traverse between the interrogated surface and the inlet into the sensor. Helpful information has been in the meantime acquired by research into the details of the method applied by dogs in their detection job [2] - and the possibilities of emulating them by fluidics. To release the detected substances from the interrogated surface, dogs use jets of exhaled breath. In the subsequent halfperiod this alternates with the sniffing inhalation phase, during which the advantage dogs possess is their ability to move their head so as to place the snout to the proper position relative to the interrogated surface. Suggested mechanical means analogous to dog's head positioning - e.g., moving pipes with end pads landing on the person's clothes - are not practically acceptable.

Perhaps least inconvenient - and already accepted by the general public - is locating the detectors in portals (Fig. 1) to be walked through. The versions currently in use have simple nozzles generating air jets directed at particular locations on the screened person. Unfortunately, such simple jets do not handle the samples properly. They blow them away from the detection position and let them escape into the atmosphere. Attempts at improvement increase the sample amount by increasing the aerodynamic power supplied to the nozzle - without real success. The jets, powerful to the level of unpleasantness, do release more substances but let them escape. In portals nowadays available [3] the collectors taking the sample into the analyser are positioned in the lintel top beam of the portal. The transport of the sample to the collector relies on thermal convection currents. Unfortunately, these currents generated by body heat are slow so that the screened person cannot simply walk through the portal at a normal pace. Also, they tend to be unreliable since the slow currents may be easily diverted by 


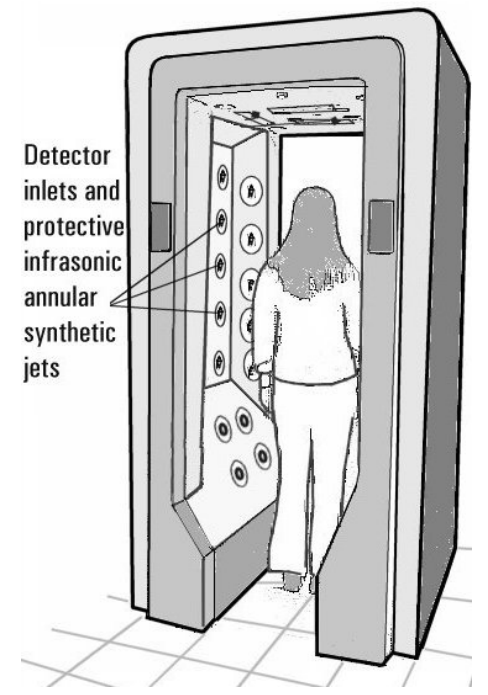

Figure 1: An example of a portal for detecting minute traces of illegal substances on a person's clothing.

unpredictable horizontal air flows (e.g. room draughts or person's motion). Not answered is the dilution of the sample by mixing with the surrounding air.

The collector inlets should instead be placed near the sample releasing nozzles and the sample has to be driven into them by another, preferably aerodynamic mechanism - e.g. a horizontal air flow. The problem of how to generate this flow is made more difficult by the inevitable separation distance to be travelled by air flows between the person's clothes and the portal side wall.

Anthropometric statistics say [4] that an acceptably small percentage - a mere $5 \%$ - of men in the USA have a shoulder breadth more than $510 \mathrm{~mm}$ while the minimum distance is defined by $5 \%$ of Hong Kong women who have their shoulder breadth less than $355 \mathrm{~mm}$. Air flows generated in the portal have to travel the difference distance between these values. It is estimated that distance $155 \mathrm{~mm}$ on each side of the portal will be able to handle $90 \%$ of the grown-up population. The remaining extreme cases - as well as children and pet animals should be handled by auxiliary detectors hand-held by attendants, which have to be present anyway to supervise the screening.

\section{Failure of steady-flow annular jet}

There seemed to be a solution meeting the requirements: the annular impinging jet (as shown in Fig. 2). The impact on the stagnation circle on the investigated surface would generate the requested releasing of the sample. Simultaneously, the return flow from the centripetal stagnation point (on jet axis) would provide the sample transport to the detector inlet. There was a belief that this flowfield will form an aerodynamically impenetrable and yet mechanically unobtrusive barrier, preventing sample dilution by mixing with external atmospheric air. 


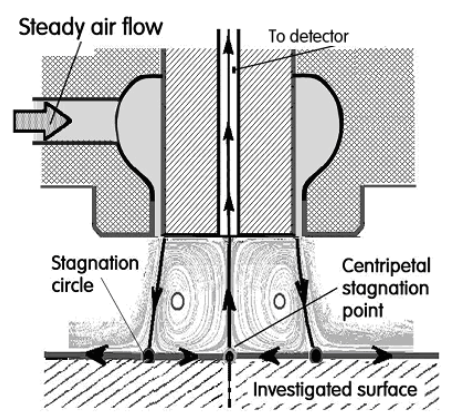

Figure 2: Unsuccessful annular air jet. The tasks to perform are: 1) agitating the surface and release the sample; 2) generating a flow from surface to detector inlet; 3 ) protecting this flow from escaping and diluting. The failure occurred whenever the nozzle-to-surface distance was more than $\sim 3$ nozzle exit diameters.

Unfortunately, attempts at implementing this idea were unsuccessful. A nozzle of reasonable diameter cannot generate an annular jet reaching far enough - not so much because of the loss of jet momentum but mainly due to the coalescence into the stagnation point $S$ terminating the annular character (Fig. 3(a)). The change of the centripetal surface flow into the centrifugal pattern is related to the metamorphosis phenomenon in [5]. Computation results,

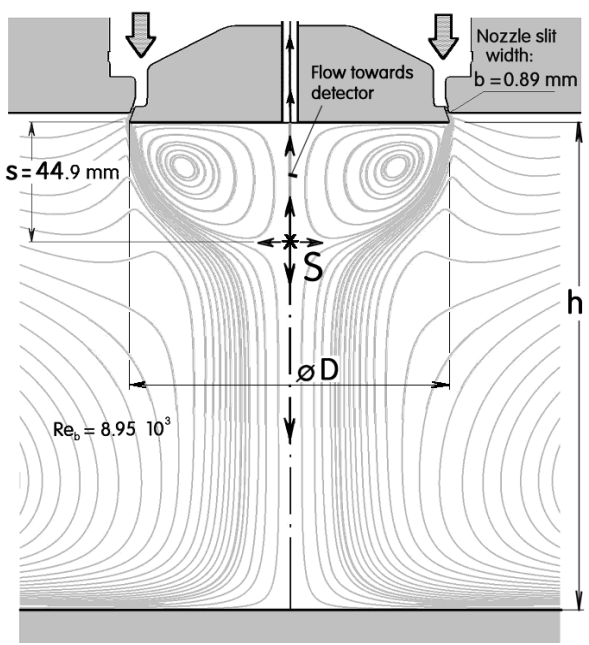

(a)

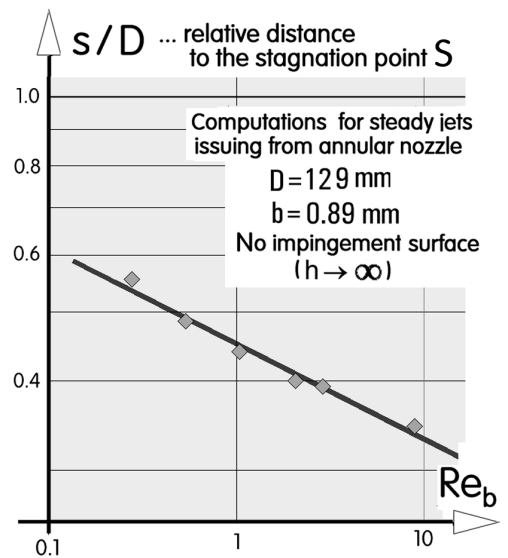

(b)

Figure 3: (a) Computed pathlines of steady annular show the loss of annular character. The recirculation region reaches only to the point $S$ and not to the surface. (b) Computed distances $S$ of the stagnation point $\mathrm{S}$ from the nozzle. Very low Reynolds numbers $\mathrm{Re}_{\mathrm{b}}$ may seem to be helpful but are impractical. 
like those in Figs. 3(a) and (b), have shown that obtaining the stagnation point at the requested distance $155 \mathrm{~mm}$ from the nozzle (Sect. 2) - with reasonable dimensions of nozzle outer diameter $D$ and slit width $b$ - is out of the question. Admittedly, the results in Fig. 3(b) apply to $h \rightarrow \infty$, i.e. absence of impingement wall with which the $s / D$ ratio increases somewhat, but the increase is much less than what is needed. Experiments have also shown problems with hysteresis [16].

\section{New idea: protective annular synthetic jet}

Synthetic jets are generated by fluidic rectification effect [7-9] from an alternating outflow and inflow through a nozzle [10]. They consist - at least over a certain distance from the nozzle [18] - of successive pairs of individual vortices. The jet is "synthesised" from them. In the unusual annular nozzle case they were first proposed for the discussed detector application in [17]. Another unusual feature of this proposal is generation of the periodic flow by means of a no-moving-part fluidic oscillator. The device thus becomes simple (Fig. 7(a)) and reliable. The generated jet is of the hybrid-synthetic jet character $[11,12]$, i.e. with non-zero time-mean flow in the nozzle. This is an important factor, helpful to generating the overall flowfield character similar to Fig. 2. The character of consisting of individual vortices make them capable of reaching very far without the coalescence, because the vortices are radially pressurebalanced already at their formation and thus their motion trajectory, as was observed in visualisations $[9,10]$, is not prone to the coalescing effect. Another factor of importance for the discussed application is the low-frequency pulsatile character (as opposed to high-frequency turbulence) promoting the release of the illicit material traces from the screened textile surface.

Existing studies of synthetic jets are aimed at control of fluid flow past bodies, usually by generation of streamwise vortices [19]. This use calls for a rather high driving frequency [20]. In the discussed application, however, operation in audible frequency range would cause inconvenience. To avoid it, the device is operated at an inaudible infrasonic [14] frequency, in practice $\leq 20 \mathrm{~Hz}$. Another unusual feature is the attempt to increase the reach of the jet by inclining the nozzle exit direction away from the nozzle axis and centrebody protruding above the outer part of the annular nozzle.

To verify the assumptions, hot wire anemometer measurements were performed using laboratory model (Figs 4(a) and (b)) together with numerical unsteady flowfield computations. Because of the large number of unknowns, it was decided to perform separate experiments with the annular synthetic jet which was for this purpose generated by a loudspeaker (Fig. 4(a)) - and with the infrasonic oscillator. In both cases the flows were studied by means of hot-wire anemometer. Interpretation of anemometer data is somewhat complicated by their inability to discriminate between the flow direction out from the nozzle and back into it. Avoiding inconvenience by operation at infrasonic frequencies has led to question whether the low frequency would not lead to extremely poor performance. In one of the earliest experiments, the probe was traversed at a 
short distance from the nozzle to measure the velocity profile at various driving frequencies from $5 \mathrm{~Hz}$ to $65 \mathrm{~Hz}$ (Figs 6(a) and 6(b)). It was established that at the frequency $20 \mathrm{~Hz}$ and with electric input power only $4 \mathrm{~W}$ the actuator did operate quite efficiently (improvement of the efficiency was not substantial at higher frequencies - Fig. 6(b)) and did not generate an audible disturbance.

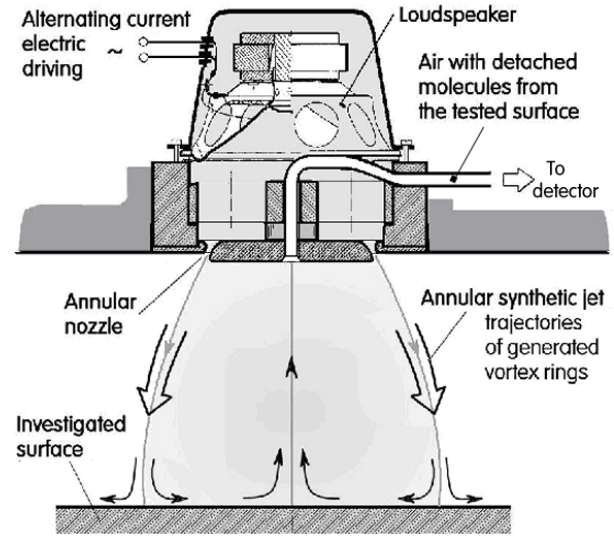

(a)

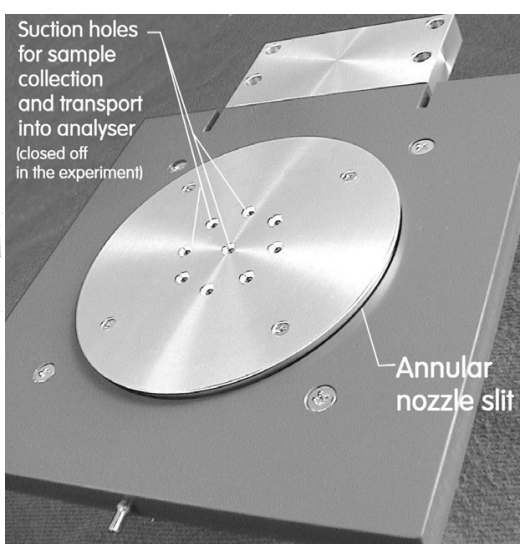

(b)

Figure 4: (a) Solution: synthetic annular jet - here in the configuration for laboratory tests, driven by a loudspeaker. The alternating outflow and inflow generates a system of vortex rings moving towards the interrogated surface. (b) Photograph of the actuator model with $129 \mathrm{~mm}$ nozzle diameter. Note the centrebody protruding slightly $(4.15 \mathrm{~mm})$ above the nozzle slit, which is inclined outwards.

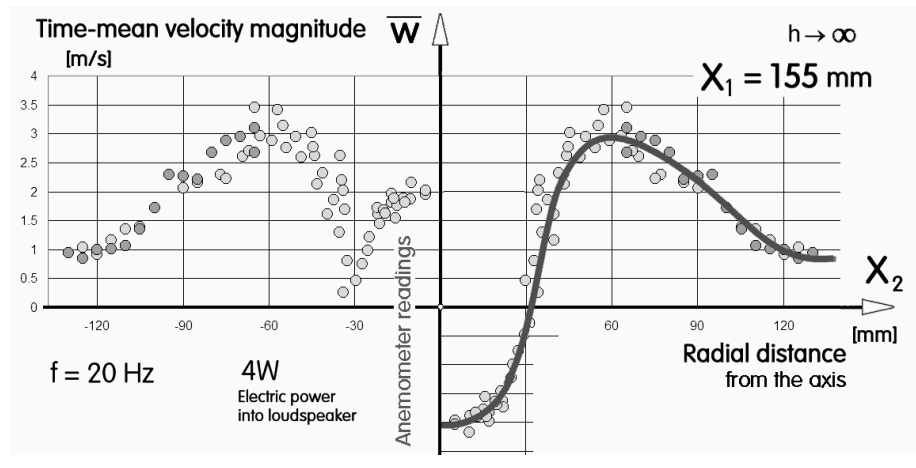

(a)

(b)

Figure 5: Time-mean velocity profile at $\mathrm{X}_{1}=105 \mathrm{~mm}$ axial distance from the nozzle: (a) absolute values measured by hot-wire anemometer; (b) fit to data with correct sign. Velocity maxima at a quite large radial distance $\mathrm{X}_{2}$ show lack of coalescence. 


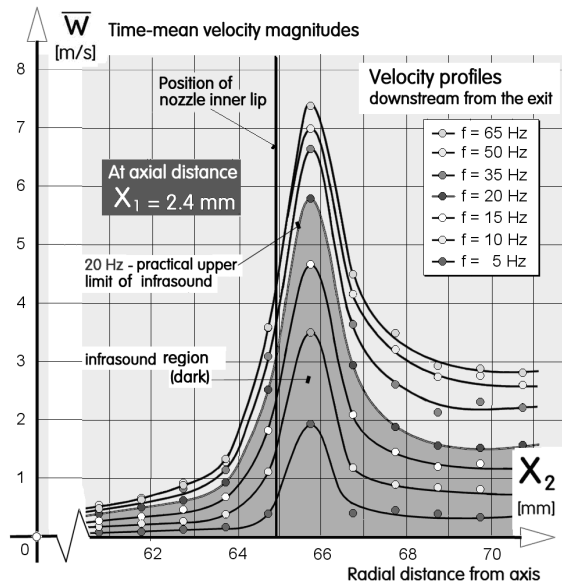

(a)

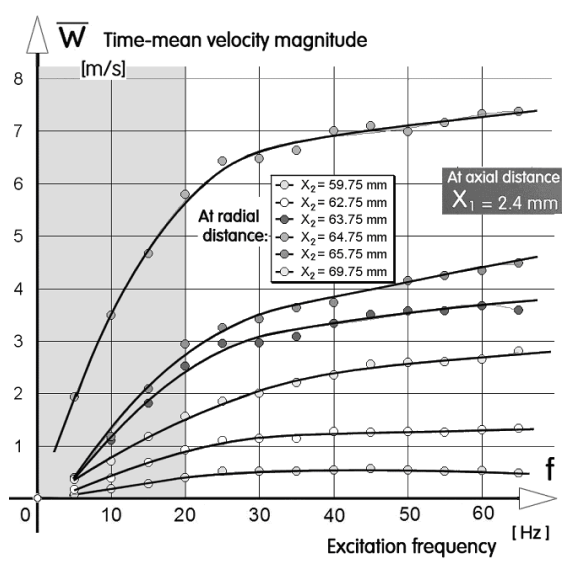

(b)

Figure 6: (a) Profiles of time-mean velocity at a small axial distance $\mathrm{X}_{1}=$ $2.4 \mathrm{~mm}$ obtained at different driving frequencies (with constant loudspeaker driving power $4 \mathrm{~W}$ ). The requirement of inaudible noise limits the operation to the shaded region; (b) Dependence of velocity peak (a) on frequency. Beyond the infrasonic $20 \mathrm{~Hz}$, the velocity growth with frequency is much slower.

The fundamental factor is the size of the protected region occupied by the stationary vortex ring in front of the nozzle. Measured velocity profiles, such as their typical example presented in Fig. 5, have shown that the boundary of the protected volume - which is coincident with the loci of velocity maxima in the profiles - indeed does extend far beyond the requirements. Recognisable velocity maxima were measured at axial distances from the nozzle more than $\mathrm{X}_{1}$ $=155 \mathrm{~mm}$. The non-zero velocity on the axis (presented as negative in Fig. 5 to account for the probe inability to indicate sign change) is actually indicative of the presence of the flow from the investigated surface towards the detector inlet.

\section{Infrasonic oscillator}

Fluidic oscillators are mostly designed by providing a fluidic amplifier with feedback loops. This traditional setup ceases to be useful if the task is to generate oscillation at very low, infrasonic frequency - which is requested in the discussed anti-terrorist application to avoid the screened persons' exposure to the annoying acoustic signal. Low frequency means long feedback channels which may be difficult to stow and the hydraulic losses inside them may be so strong that the feedback signal reaches the amplifier input at a too weak level.

The oscillator tested in the course of developing the anti-terrorist detector is therefore based on a wholly new idea. There is a vestigial fluidic jet-deflection diverter amplifier, which does not possess any control nozzle, being switched by the load-switching mechanism. Instead of the travelling-time delay in a long 


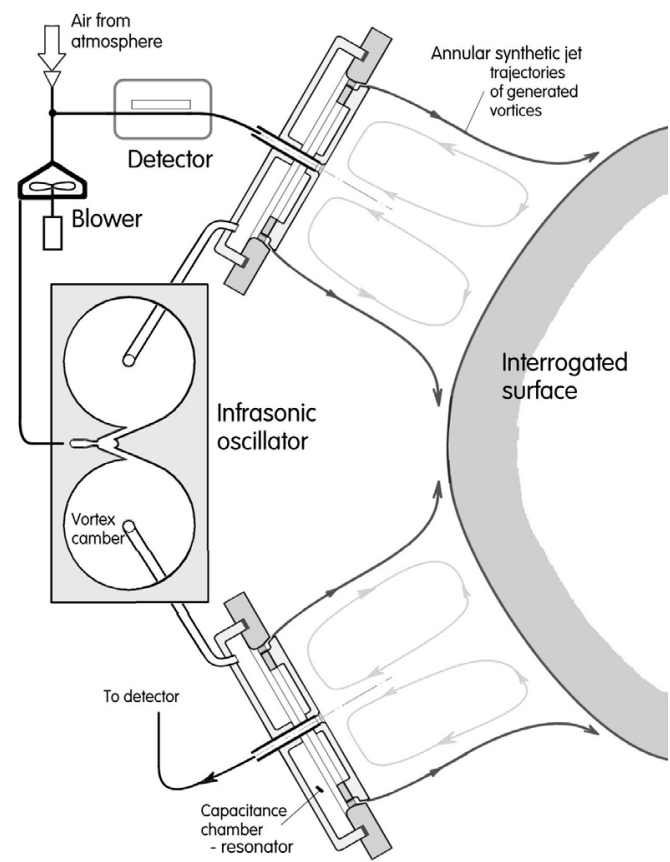

(a)

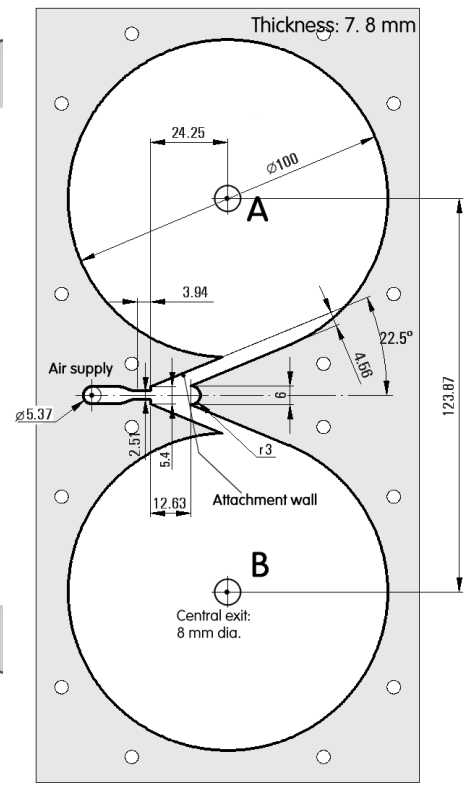

(b)

Figure 7: (a) Schematic representation of two actuators generating two annular two synthetic jets driven by common infrasonic fluidic oscillator; (b) Cavities of the fluidic oscillator model as used in the feasibility tests.

feedback channel, the low frequency is achieved by using the spin-up time in vortex chambers.

A drawing (with dimensions) of the oscillator used in the feasibility testing is presented in Fig. 7(b). Cavities were made by laser-cutting in a $7.8 \mathrm{~mm}$ thick plate of PMMA. Air from the supply inlet (diameter $5.37 \mathrm{~mm}$ ) is lead into the nozzle (of $2.51 \mathrm{~mm}$ width) from which it issues as an air jet between two attachment walls (mutually inclined by $45 \mathrm{deg}$ ). The jet attaches to one of them by the Coanda effect and is led into the corresponding vortex chamber. Let us assume it enters tangentially the upper vortex chamber $A$, generating there a rotational flow governed by the law of conserving the moment of momentum. This means that as fluid goes to the central exit and the radius of rotation gradually decreases, the local rotational speed gradually increases. In the end it attains a magnitude so high that the resultant centrifugal force opposes the input flow into chamber A so strongly that it cannot be any more overcome by the Coanda effect. The air jet separates from the attachment wall and is switched to the other one, leading into the vortex chamber B, where the rotation has in the meantime stopped. After the switching, the rotation in the chamber B increases 
in intensity. It finally attains a magnitude so high that the resultant centrifugal force opposes the input flow into the chamber B so strongly that it cannot be any more overcome by the Coanda effect and the air jet is switched back.

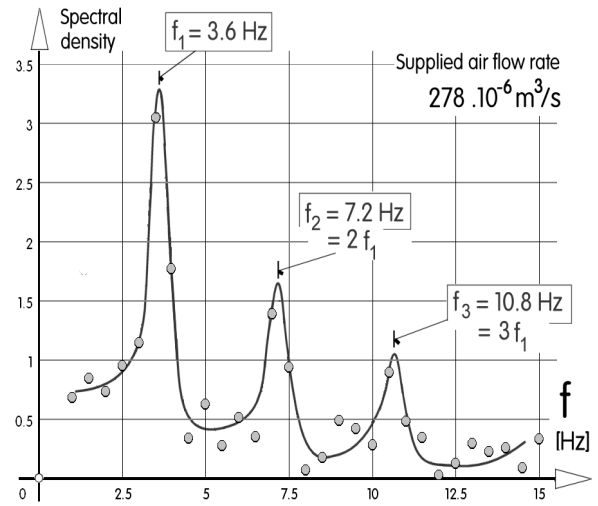

(a)

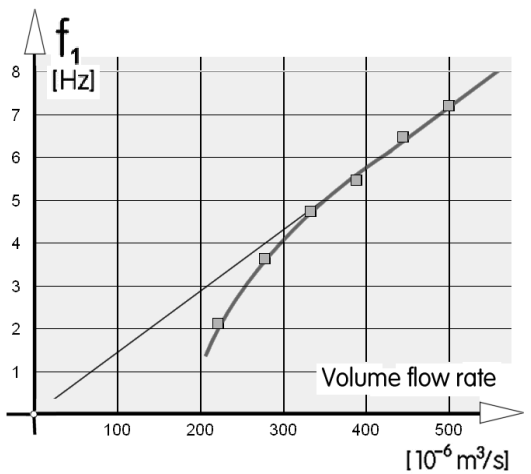

(b)

Figure 8: (a) An example of oscillator frequency spectrum measured by hotwire anemometer in one of the two output terminals; (b) Dependence of the dominant frequency $f_{1}$ (cf. Fig. 8(a)) on the supplied air flow into the oscillator model.

The oscillation frequency values seen in Fig. 8(b) are very low, indeed in the infrasonic range. In Fig. 8(a) is a spectrum of the output signal measured in one of the oscillator's two output terminals by a hot-wire anemometer. There are in this spectrum three local peaks of which the one at the lowest frequency $f_{1}$ is clearly dominant. The other two smaller peaks are at frequencies which are integer multiples. As usual in most fluidic oscillators, the frequency tends to be proportional to the supplied flow rate, i.e. there is a constant Strouhal number. Included in Fig. 8(b) are two data points obtained by measurements at very low flow rates, at which there are some friction effect causing a decrease of the Strouhal number - again a phenomenon known from other fluidic oscillators.

\section{Conclusions}

Annual synthetic jets were demonstrated both experimentally and by numerical solutions to fulfil the requirements - especially the long axial reach towards the interrogated surface -requested for capability to transport trace substance samples from the surface to the entrance into the analyser. The closed boundaries of the active region prevent dilution by external atmospheric air and the pulsatory character helps in detachment of the sample. The low frequency makes the device practically inaudible. 


\section{Acknowledgements}

Gratefully acknowledged is institutional support RVO: 61388998 and also support by grant Nr.23-046S from the GAČR - Czech Science Foundation.

\section{References}

[1] Jain, S., Mukand, W. (2004) The economics of high-visibility terrorism, European Journal of Political Economy, Vol. 20, p. 479

[2] Tesař V. (2007) Pressure-Driven Microfluidics. Artech House Publishers, Boston - London

[3] Settles G. S. (2006) Fluid mechanics and homeland security, Annual Review of Fluid Mechanics, Vol. 38, p. 87

[4] Ruff C. (2002) Variation in Human Body Size and Shape, Annual Review of Anthropology, Vol. 31, p. 211

[5] Tesař V., Trávníček Z. (2008) Excitational metamorphosis of surface flowfield under an impinging annular jet, Chemical Engineering Journ., Vol. 144, p. 312

[6] Tesař V., Trávníček Z. (2006) Apparatus for collection of samples from the surface of examined objects, in Czech language, Patent Application No. PV 2006-214, Patent Office of the Czech Republic

[7] Tesař V. (1984) Law governing entrainment of surrounding fluid during alternating inflow into and outflow from an orifice - in Czech, Czechoslovak Patent Office Application PO 86-84, Prague, Czech Republic

[8] Tesař V., Zhong S.(2003) Efficiency of synthetic jet generation, Transactions of the Aeronautical and Astronautical Society of the Republic of China, Zhongguo Hangkong Taikong Xuehui Huikan, Vol. 35, No.1, p. 45

[9] Tesař V., Trávníček Z. (2005) Pulsating and Synthetic Impinging Jets, Journal of Visualization, Vol. 8, No. 3, p. 201

[10] Trávníček Z., Tesař V. (2003) Annular synthetic jet used for impinging flow mass transfer, Internat. Journ. of Heat and Mass Transfer, Vol. 46, p. 3291

[11] Tesař V. (2007) Configurations of fluidic actuators for generating hybridsynthetic jets, Sensors and Actuators A: Physical, Vol. 138, p. 394

[12] Trávníček Z., Vít T., Tesař V. (2006) Hybrid synthetic jets as the nonzeronet-mass-flux synthetic jets, Physics of Fluids, Vol. 18, p. 081701-1

[13] Tesař V., Hung C.-H., Zimmerman W. (2006) No-moving-part hybridsynthetic jet actuator, Sensors and Actuators A: Physical, Vol. 125, p. 159

[14] Gavreau V. (1968), Infrasound, Science Journal, Vol. 4, Issue 4, p. 33

[15] Tesař V., Kordík J. (2013), Effective hydraulic resistance of a nozzle in an electrodynamic actuator generating hybrid-synthetic jet - Parts I and II, Sensors and Actuators: A Physical, Vol. 199, p. 379, 391

[16] Trávníček Z., Tesař V. (2013), Hysteresis in annular impinging jets, Experimental Thermal and Fluids Science, Vol. 44, p. 565

[17] Tesař V. (2014), Fluidic oscillator, Czech Patent Appl. PV 2014-903, Dec. 2014 
[18] Tesař V., Kordík J. (2010), Time-Mean Structure of Axisymmetric Synthetic Jets, Sensors and Actuators: A. Physical, Vol. 161, p. 217

[19] Tesař V., Kordík J., Daněk M. (2008) Lift and Separation Control on Wind Turbine Blades by Vortices Having Streamwise Oriented Axes, Proc. of "Fluid Dynamics 2008”, ISBN 978-80-87012-14-7, Inst. of Thermomechanics AS CR, v.v.i., Prague

[20] Tesař V., Zhong S., Fayaz R. (2013) New fluidic oscillator concept for flow separation control, AIAA Journal, Vol. 51, p. 397 